\title{
Compact Balanced FSIR Bandpass Filter Modified for Enhancing Common-Mode Suppression
}

\author{
Armando Fernández-Prieto, Jesús Martel, Member, IEEE, Francisco Medina, Fellow, IEEE, \\ Francisco Mesa, Fellow, IEEE, and Rafael R. Boix, Member, IEEE
}

\begin{abstract}
A novel balanced bandpass filter (BPF) based on folded stepped impedance resonators (FSIR's) with modified ground plane is presented in this work. By symmetrically introducing a series-LC resonant structure below the FSIR's, common-mode (CM) propagation can be rejected without affecting differential-mode (DM) performance. The filter presents two main advantages with respect to the conventional solid-ground-plane FSIR filter: i) an important improvement of the CM rejection level within the differential passband and ii) an enhanced filter selectivity due to the inclusion of an extra transmission zero in the differential passband. Both the conventional and the novel filters have been modeled as lumped-element circuits that fully account for DM and CM operation. Simulation and measurement results confirm the benefits of the proposed balanced BPF.
\end{abstract}

Index Terms-Balanced filter, common-mode (CM) suppression, double-side MIC technology, defected ground structures (DGS).

\section{INTRODUCTION}

$\mathbf{N}$ OWADAYS, many approaches are available to deal with the problem of common-mode (CM) noise suppression in $\mathrm{GHz}$ differential filters. In microstrip technology, some reported solutions are based on the use of synthetic differential lines with some type of defected ground structure (DGS), which might be periodically etched below the differential-signal coupled pair [1]-[7]. With this procedure, an all-pass behavior is ideally obtained for the differential mode (DM) while a stop band is achieved for CM signal. The differential-lines proposed in [1]-[7] are intended to be used as input/output lines of a differential filter, which leads to an increase in filter size. If size is a relevant issue, it is more convenient to design filters that simultaneously fulfill specifications for both the DM and the CM. Coupled-line filters [8], coupled resonator filters [9]-[11] or open-split-ring/complementary-split-ring resonators filters [12] are a few examples of balanced bandpass filters (BPFs) with good intrinsic CM rejection. In this letter, a novel and compact

Manuscript received September 24, 2014; accepted December 28, 2014. Date of publication January 22, 2015; date of current version March 09, 2015. This work was supported by the Spanish Ministry of Economy and Competitiveness and European Union Feder Funds under contracts TEC2013-41913-P and Consolider EMET CSD2008-00066 and by the Spanish Junta de Andalucía (project P12-TIC-1435).

A. Fernández-Prieto, F. Medina, and R. R. Boix are with the Department of Electronics and Electromagnetism, Faculty of Physics, University of Sevilla, Seville 41012, Spain (e-mail: armandof@us.es; medina@us.es).

J. Martel is with the Department of Applied Physics II, ETSA, Seville 41012, Spain.

F. Mesa is with the Department of Applied Physics I, ETSII, Seville 41012 Spain.

Color versions of one or more of the figures in this paper are available online at http://ieeexplore.ieee.org.

Digital Object Identifier 10.1109/LMWC.2015.2390492 balanced BPF based on a modified version of the electrically coupled folded stepped-impedance resonators (FSIR's) is presented together with its design procedure. Experimental validation of the proposed structure is provided through a specific design.

\section{Proposed Structure And ExPerimental Results}

The layout of the proposed balanced BPF is shown in Fig. 1. Two electrically coupled FSIR's printed on the top plane of the substrate are capacitively coupled to two symmetrically placed series-LC resonators etched in the ground plane. The design process starts by designing a conventional (with solid ground plane) FSIR filter using the standard procedure [13]. These are the DM specifications: Butterworth $N=2$, center frequency $f_{0}=2.5 \mathrm{GHz}$, and fractional bandwidth $\Delta=10 \%$. The substrate parameters are $\varepsilon_{\mathrm{r}}=5.9$ and thickness $h=0.508 \mathrm{~mm}$. FSIR dimensions have been chosen to provide a first resonance (odd symmetry) frequency at $f_{0}=2.5 \mathrm{GHz}$. The dimensions (in $\mathrm{mm}$ ) that satisfy this condition are: $l_{1}=4.75, l_{2}=4.71$, $l_{3}=2.81, l_{4}=3.83, w_{0}=0.75, w_{1}=0.6, s_{1}=0.2$ and $t=2.7$. Equivalent circuits can be extracted for the $\mathrm{CM}$ and DM, as shown in Fig. 2. There is a clear connection between the circuit parameters and the numbered sections in Fig. 1(a). Thus, $L_{1}$ is the inductance associated with Section 1 (and 6): it is a short-ended line for the DM. Conversely, $C_{1}$ is the capacitance of those sections for the CM (AA' is a virtual-open in that case). The remaining parameters are obviously the same for DM and CM: $L_{2}$ is the inductance of Sections 2 and $5, C_{2}$ is the coupling capacitance between Sections 3 and 4 , and $C_{3}$ is the capacitance between Sections 3 and 4 and the ground plane. The values of the elements have been analytically approximated (each parameter represents an electrically short transmission line section) and then slightly tuned with the help of Agilent ADS circuit simulator. The full-wave simulation results for this filter together to its $\mathrm{CM}$ and DM equivalent-circuit responses plotted in Fig. 3 show a reasonably good agreement. It can be observed a relatively poor CM rejection (around $20 \mathrm{~dB}$ within the differential passband) as well as poor symmetry of the differential passband.

The next step in the design process is the introduction of two series-LC resonant structures in the ground plane [Fig. 1(b)] to improve the $\mathrm{CM}$ rejection without increasing the filter size. New elements have to be included in the CM and DM equivalent circuits to account for the ground plane pattern (see Fig. 4). Considering that the $\mathrm{AA}^{\prime}$ plane is a virtual open for $\mathrm{CM}$ operation, the return current flows to the ground plane through the meandered lines (Sections 9 and 10), whose inductance value is represented by $L_{3}$. However, AA' behaves as a virtual short for DM operation, in such a way that the displacement current flowing between Sections 3 and 7 (or 4 and 8 ) has two different paths to flow to the ground plane: a high-impedance one (sections 9 and 

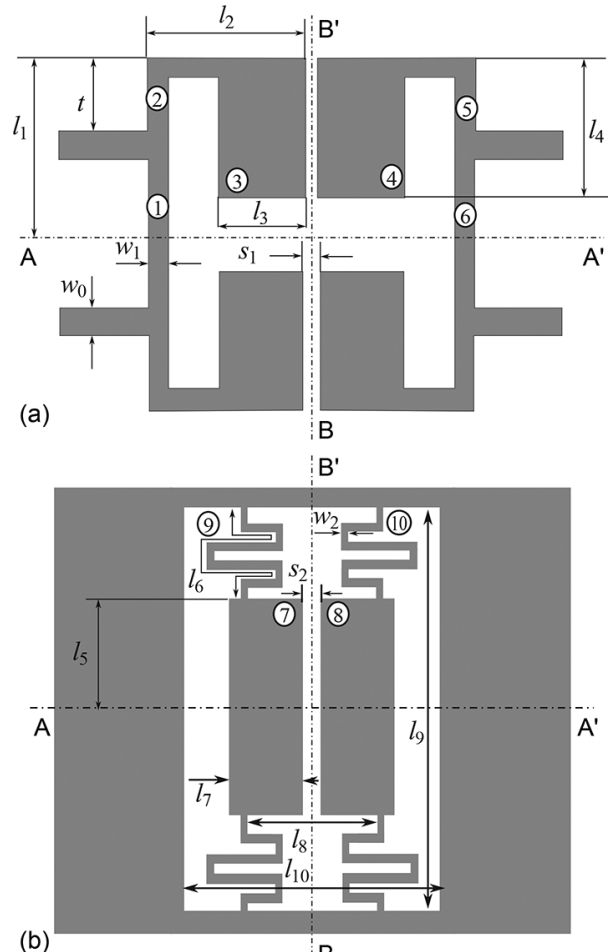

B

Fig. 1. Layout of the proposed filter. (a) Top layer metallization and (b) bottom layer metallization.

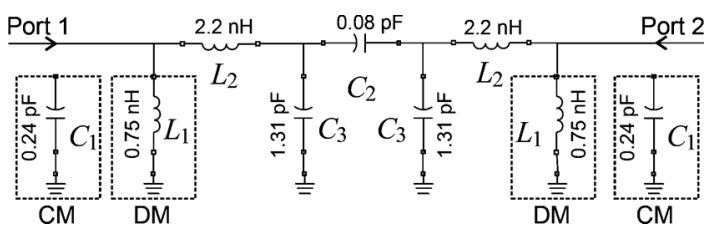

Fig. 2. Equivalent circuits for DM and CM operation of the conventional (solid ground plane) FSIR filter.

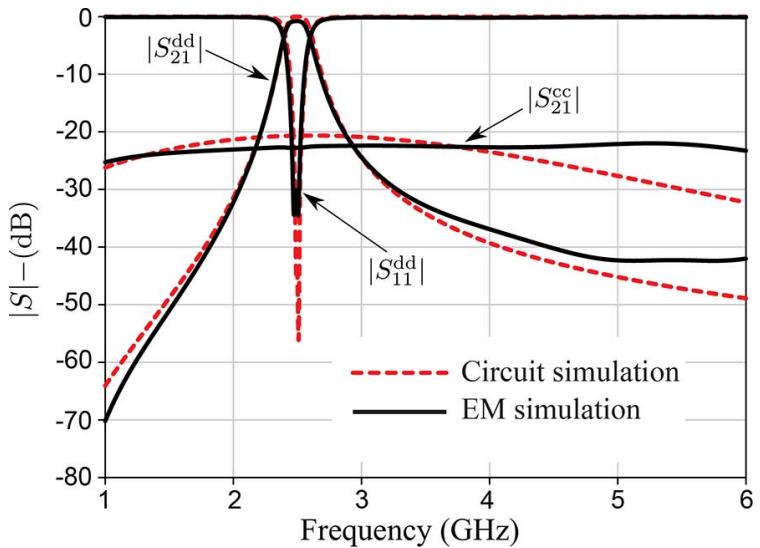

Fig. 3. Comparison between full-wave and circuit simulations for the conventional balanced BPF.

10) and a low-impedance one (sections 7 and 8). The current will then flow mainly through the low-impedance section, which is represented by a much lower value of $L_{3}$. $C_{4}$ accounts for the electric coupling between sections 7 and 8 and $M$ accounts for the magnetic coupling between sections 9 and 10 (CM operation) or 7 and 8 (DM operation). Moreover, it should be noted that, for the new filter geometry, $C_{3}$ represents the capacitance between Sections 3 and 4 and the bottom plane patches (sections 7 and 8 ). The dimensions (in $\mathrm{mm}$ ) for the new filter are:
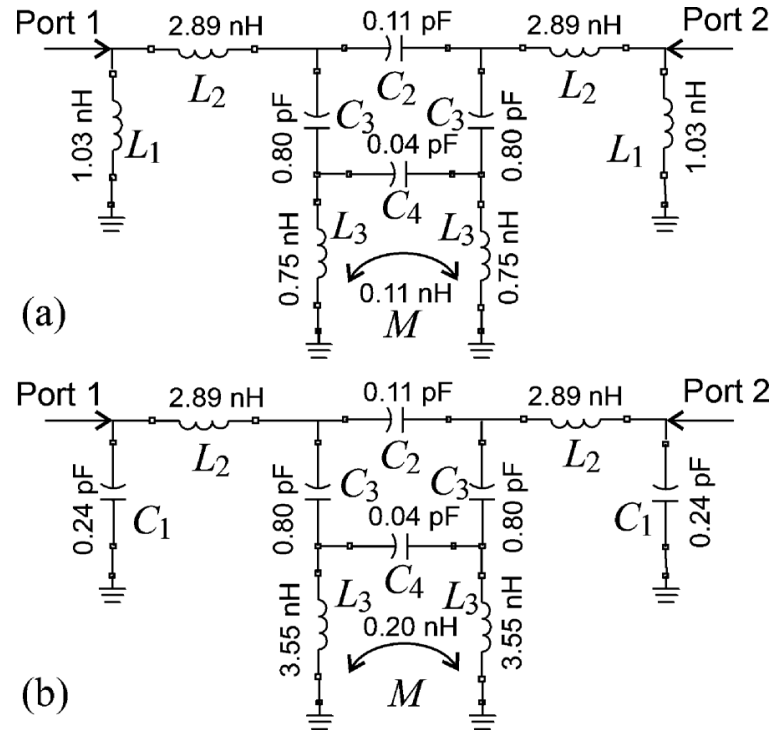

Fig. 4. Equivalent circuits of the novel filter for (a) differential-mode and (b) common-mode.

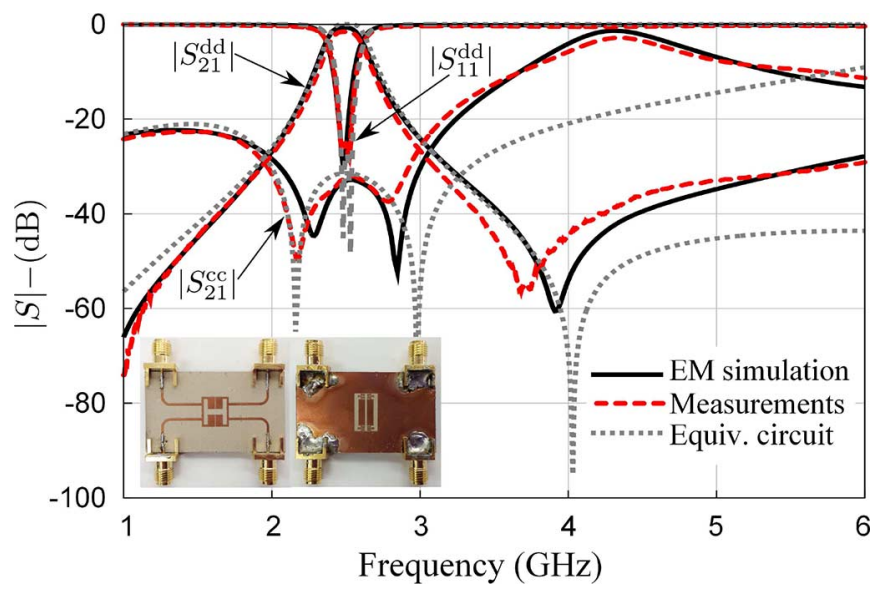

Fig. 5. Comparison between measurements, full-wave simulations and circuit simulations for the fabricated balanced BPF.

$l_{1}=5.05, l_{2}=4.21, l_{3}=2.31, l_{4}=3.83, l_{5}=4.15$, $l_{6}=7.9, l_{7}=2.11, l_{8}=2.82, l_{9}=12.1, l_{10}=7.42$, $w_{2}=0.2, t=2$ and $s_{2}=0.6$. The new set of dimensions for the FSIRs is slightly different from the one corresponding to the conventional FSIR filter in order to compensate the small perturbation introduced in the DM by the slotted ground plane.

In order to validate our proposal, the circuit whose layout is depicted in Fig. 1 has been fabricated and measured with an Agilent PNA-E8363B with a Test-Set N4420B extension. Simulated and measured DM and CM responses are shown in Fig. 5. Equivalent circuits responses are included for comparisons purposes. Thanks to the introduction of two CM TZ's, CM rejection is significantly better than for the conventional implementation. Additionally, the DM TZ observed at $1.5 f_{0}$ leads to a significant improvement of the response symmetry and selectivity. Measured insertion losses at the center frequency are $1.57 \mathrm{~dB}$. The filter size is quite small $(0.15 \lambda \times 0.21 \lambda$, with $\lambda$ being the guided wavelength at the filter center frequency). A comparison between our proposal and other contributions found in the literature is provided in Table I. According to the table, filter size, CMRR and differential-mode selectivity are found to be competitive in spite of the simplicity of the new design. 
TABLE I

COMPARISON OF SEVERAL BALANCED FILTERS

\begin{tabular}{|c|c|c|c|c|c|c|c|c|}
\hline & & \multirow{2}{*}{ Ref. } & $N$ & $\begin{array}{c}\text { Size } \\
\left(\lambda_{g}^{2}\right)\end{array}$ & $\begin{array}{c}\text { CMRR } \\
(\mathrm{dB})\end{array}$ & \multicolumn{2}{|c|}{$\begin{array}{c}S_{21}^{c c} \mid> \\
30 \mathrm{~dB}\end{array}$} & \multicolumn{4}{|c|}{ Differential mode } \\
\cline { 6 - 10 } & & & & $(\mathrm{GHz})$ & $f_{0}^{d}$ & $3-\mathrm{dB}$ & $\mathrm{IL}$ & $\Delta f_{3 \mathrm{~dB}}$ \\
\hline$[7]$ & 2 & 0.29 & 41 & $0.99 f_{0}^{d}-1.28 f_{0}^{d}$ & 2.42 & 6.6 & 0.8 & 0.32 \\
\hline$[9]$ & 2 & 0.044 & 24 & $<30 \mathrm{~dB}$ & 1.57 & 8.92 & 1 & 0.18 \\
\hline$[10]$ & 4 & 0.047 & 31 & $0.5 f_{0}^{d}-5.88 f_{0}^{d}$ & 1.02 & 12 & 3.51 & 0.52 \\
\hline$[11]$ & 4 & 0.034 & 45 & up to $5.91 f_{0}^{d}$ & 1.02 & 9.83 & 1.76 & 0.51 \\
\hline$[12]$ & 3 & 0.045 & 53 & $0.5 f_{0}^{d}-1.1 f_{0}^{d}$ & 1 & 45 & 1.2 & 0.49 \\
\hline $\begin{array}{c}\text { This } \\
\text { work }\end{array}$ & 2 & 0.031 & 34 & $0.8 f_{0}^{d}-1.2 f_{0}^{d}$ & 2.5 & 10 & 1.57 & 0.38 \\
\hline
\end{tabular}

$M(\mathrm{nH})$

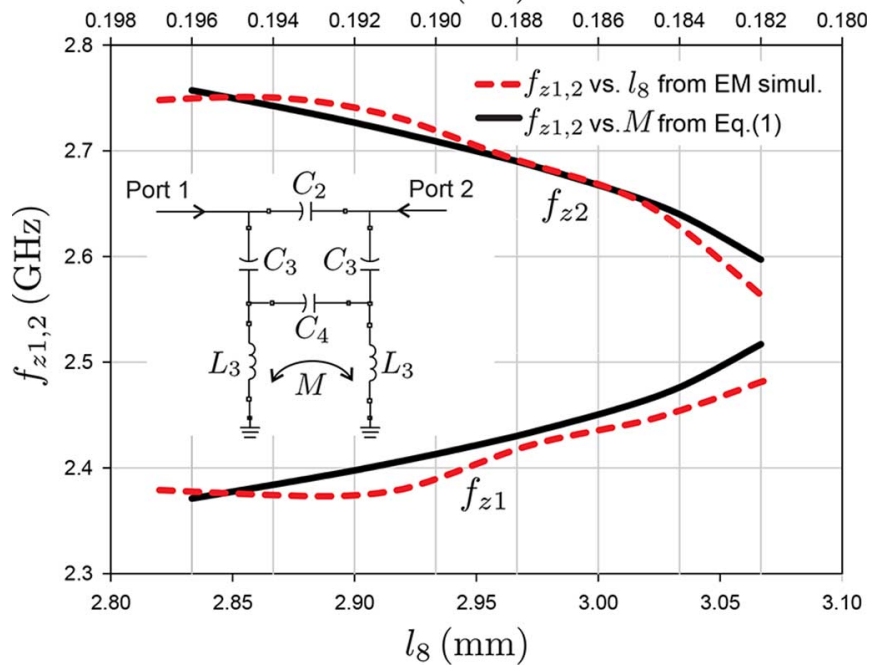

Fig. 6. (Color online) $\mathrm{CM} \mathrm{TZ} \mathrm{frequencies} \mathrm{versus} l_{8}$ (EM simulation) and $M$ (1). The inset shows the equivalent circuit section used for the calculation of the embedded TZ's.

\section{Control of the Transmission Zeros}

DM and CM TZ's are determined by the values of the circuit elements that account for the coupling between resonators as well as for the presence of the ground plane pattern (see the inset in Fig. 6). The characteristic equation providing the location of the zeros is given by

$$
\begin{aligned}
& Z_{C 3}^{2}\left(2 Z_{L 4}+Z_{C 4}\right)+2 Z_{C 3} Z_{C 4} Z_{L 4} \\
& \quad+Z_{L 4}^{2}\left(2 Z_{C 3}+Z_{C 2}+Z_{C 4}\right)+Z_{C 3} Z_{M}\left(4 Z_{L 4}+2 Z_{C 4}\right) \\
& \quad+Z_{C 2} Z_{M}\left(Z_{C 4}+Z_{L 4}\right)+2 Z_{L 4} Z_{M} Z_{C 4}=0
\end{aligned}
$$

where $L_{4}=L_{3}-M$ and $Z_{X i}$ represents the impedance of the $X_{i}$ component ( $X$ is $C$ or $L$ and $i$ is the index number, $i=2,3$ or $i=4)$. The location of the TZ's can easily be adjusted by proper selection of the values of the circuit components (and the structural dimensions that give place to those values). As an example, Fig. 6 compares the CM TZ frequencies as a function of $M$ using both (1) and numerical simulations. Note that $M$ is mainly controlled by $l_{8}$, which is varied while the remaining parameters are kept constant. Fig. 6 clearly shows that there is an apparent correlation between the geometrical parameter $l_{8}$ and the circuit parameter $M$, as expected from our circuit interpretation of the physical structure. This figure also demonstrates the suitability of (1) to control the position and bandwidth of the $\mathrm{CM}$ rejection band.

\section{CONCLUSION}

A new compact balanced BPF based on a modified version of electrically coupled FSIR's is presented. Equivalent circuits are provided to facilitate the design process of both DM and $\mathrm{CM}$ responses. The proposed structure provides a significant improvement of CM rejection level and a better DM symmetry response and selectivity when compared with the conventional FSIR filter.

\section{REFERENCES}

[1] W.-T. Liu, C.-H. Tsai, T.-W. Han, and T.-L. Wu, "An embedded common-mode suppression filter for $\mathrm{GHz}$ differential signals using periodic defected ground plane," IEEE Microw. Wireless Compon. Lett., vol. 18, no. 4, pp. 248-250, Apr. 2008.

[2] S.-J. Wu, C.-H. Tsai, T.-L. Wu, and T. Itoh, "A novel wideband common-mode suppression filter for gigahertz differential signals using coupled patterned ground structure," IEEE Trans. Microw. Theory Tech., vol. 57, no. 4, pp. 848-855, Apr. 2009.

[3] C.-H. Tsai and T.-L. Wu, "A broadband and miniaturized commonmode filter for gigahertz differential signals based on negative-permittivity metamaterials," IEEE Trans. Microw. Theory Tech., vol. 58, no. 1, pp. 195-202, Jan. 2010.

[4] J. Naqui, A. Fernández-Prieto, M. Durán-Sindreu, F. Mesa, J. Martel, F. Medina, and F. Martín, "Common mode suppression in microstrip differential lines by means of complementary split ring resonators: theory and applications," IEEE Trans. Microw. Theory Tech., vol. 60, no. 10, pp. 3023-3033, Oct. 2012.

[5] A. Fernández-Prieto, J. Martel, J. S. Hong, F. Medina, S. Qian, and F. Mesa, "Differential transmission line for common-mode suppression using double side MIC technology," in Proc. 41st Eur. Microw. Conf. (EuMC), Manchester, U.K., Oct. 10-13, 2011, pp. 631-634.

[6] A. Fernández-Prieto, J. Martel, F. Medina, F. Mesa, S. Qian, J.-S. Hong, J. Naqui, and F. Martín, "Dual-band differential filter using broadband common-mode rejection artificial transmission line," Prog. Electromag. Research (PIER), vol. 139, pp. 779-797, Apr. 2013.

[7] H. Wang, K.-W. Tam, S.-K. Ho, W. Kang, and W. Wu, "Short-ended self-coupled ring resonator and its application for balanced filter design," IEEE Microw. Wireless Compon. Lett., vol. 25, no. 5, pp. 312-314, May 2014.

[8] C.-H. Wu, C.-H. Wang, and C. H. Chen, "Novel balanced coupled-line bandpass filters with common-mode noise suppression," IEEE Trans. Microw. Theory Tech., vol. 55, no. 2, pp. 287-295, Feb. 2007.

[9] J. Shi and Q. Xue, "Balanced bandpass filters using center-loaded halfwavelength resonators," IEEE Trans. Microw. Theory Tech., vol. 58, no. 4, pp. 970-977, Apr. 2010.

[10] C.-H. Wu, C.-H. Wang, and C.-H. Chen, "Stopband-extended balanced bandpass filter using coupled stepped-impedance resonators," IEEE Microw. Wireless Compon. Lett., vol. 17, no. 7, pp. 507-509, Jul. 2007.

[11] J.-L. Olvera-Cervantes and A. Corona-Chavez, "Microstrip balanced bandpass filter with compact size, extended-stopband and commonmode noise suppression," IEEE Microw. Wireless Compon. Lett., vol. 23 , no. 10 , pp. 530-532, Oct. 2013

[12] P. Vélez, J. Naqui, A. Fernández-Prieto, M. Durán-Sindreu, J. Bonache, J. Martel, F. Medina, and F. Martín, "Differential bandpass filter with common-mode suppression based on open split ring resonators and open complementary split ring resonators," IEEE Microw. Wireless Compon. Lett., vol. 23, no. 1, pp. 22-24, Jan. 2013.

[13] J.-S. Hong, Microstrip Filters for RF/Microwave Applications, 2nd ed. New York: Wiley, 2011, ch. 7. 